\title{
RECOVERY OF GEOGRAPHIC ENTITIES IN TEXT FOR THE ANALYSIS OF SPATIAL PATTERNS OF ROAD INCIDENTS
}

\author{
ALEXANDER ROBLES M., CÉSAR A. PERDOMO CH \& JULí́N R. CAMARgO L
}

Engineering Faculty, Universidad Distrital Francisco José de Caldas, Bogotá, Colombia

\begin{abstract}
This document shows the use of unstructured information from open collaboration systems to extract geographical context and transform it into useful spatial data for the analysis of road incident patterns in the city of Bogotá. In this case, computational intelligence techniques have been used to handle the problem of text classification with natural language processing and the information extraction methodology "Named Entity Extraction".

KEYWORDS: Gazetteers, Geocoding, Natural Language Processing, Spatial Data, Spatial Analysis, Vector-Support Machines
\end{abstract}

Received: Jun 08, 2020; Accepted: Jun 28, 2020; Published: Oct 10, 2020; Paper Id.: IJMPERDJUN20201501

\section{INTRODUCTION}

Much of the information generated today is unstructured information and is found in text form on the web, mostly shared in open collaboration systems, for example, in social networks, blogs, articles, among others. The texts that are shared have different motivations and some inform the community about events that are likely to be georeferenced, this is the case of events on the urban road network that generates congestion. Congestion in road networks represents one of the greatest challenges because it behaves in different ways depending on the spatial location, so it has been established then divisions by behavior and cause, non-recurring traffic focuses on-road incidents that cause congestion and recurring traffic refers to regular patterns that generally occur in a specific site, road or fixed area during a period that usually corresponds to peak hours, is a common occurrence due to high demand for transport, insufficient traffic capacity or poor infrastructure. Recurrent traffic affects the city on a regional scale, so an evolutionary pattern is expected. Identifying the evolutionary pattern allows the design of traffic improvement strategies that benefit the mobility of the city.

The monitoring of road congestion is an important problem and a challenge for state entities, the main way to do this control requires the development of complex sensor structures positioned in some strategic points of cities, these installations in addition to limiting the spatial coverage of control is costly, therefore its accuracy is limited [1], thus the use of text information in social networks becomes an alternative to low-cost data. This document describes the process of retrieval of geographic information or geographic context of a text to locate road incidents and identify spatial patterns in the data.

This paper is organized into sections. Section 2 corresponds to the selection of the natural processing algorithm of the language that best behaves before classification. Section 3 shows the study area spatially, 4 shows a contextualization of how data are normally obtained in the problem posed, Section 5 refers to the training of the vector support machine and emphasizes the search for hyperparameters of the model, Section 6 deals with the 
methodology "NaMED Entity Extraction" (NER) and "Geographic information retrieval" (GIR) with the implementation for the extraction of directions at a point scale. Geocoding is dealt with in section 7 together with the identification of spatial patterns, finally, the conclusions are dealt with in section 8 , it is related to future developments and work on the topic of geographic information retrieval.

\section{NLP AND TEXT CLASSIFICATION ALGORITHM}

Natural language is far from a programming language because it is not intended to be translated into a finite number of mathematical operations, this is mainly because natural language is used to share information among human beings, i.e. a programming language tells the computer exactly what it should do, but there are no compilers for languages such as English, Spanish or French.

So natural language processing (NLP) is an area of research in computer science and artificial intelligence (AI) that relates to the processing of natural languages such as English or Spanish, that process involves translating natural language into numbers that a computer can understand, classify or use in the generation of new text [2]. In natural language processing, the same tasks that human beings do daily are performed with weighting, fragmentation, voice tagging, automatic translation or voice recognition, all of which represent a great computational challenge.

The information age made it necessary to understand not only structured data but also unstructured data such as language, this represents a challenge for industries as we generate petabytes of information in blogs, tweets, Facebook states, chats, emails and comments on the web, this information is collected to improve the customer experience and open business niches, currently, there are many fields of application of language processing techniques, within which they are [3]:

- Search Engines

- Voice Search Engines

- Mail sorters

- Translators

Classification is a fundamental problem in natural language processing and there are numerous classification applications such as document organizers, news filtering, spam detection, opinion mining and sentiment identification. This can be achieved through traditional methods such as dictionaries of search words in a text that allow categorization. Recently in these classification tasks are found deep learning models that include convolutional neural networks (CNN) and recurrent neural networks (RNN), these models of deep learning prioritize locality and sequentially, in addition to capturing semantic and syntactic information in sequences of consecutive words.

The way to analyze this data is to transform the text into a vector form, this is called "term-document matrix" and its function is to measure the frequency with which a word is repeated in a string of characters, this can be seen in Table 1.

Table 1: Text vectorization

\begin{tabular}{|l|l|l|l|l|l|l|l|l|l|l|l|l|}
\hline TDM & Any more & call & camera & color & cried & enough & entitled & free & gon & had & latest & mobile \\
\hline SMS1 & 0 & 1 & 1 & 1 & 0 & 0 & 1 & 2 & 0 & 1 & 0 & 3 \\
\hline
\end{tabular}




\begin{tabular}{|l|l|l|l|l|l|l|l|l|l|l|l|l|l|}
\hline SMS2 & 0 & 0 & 0 & 0 & 1 & 1 & 0 & 0 & 1 & 0 & 0 & 0 \\
\hline
\end{tabular}

Under this vectorial representation and accompanied by a Boolean variable that classifies a text, for example: road incident (1) and no road incident (0). In this format, the organized data is obtained to be approached as any automatic learning problem, where it is possible to subdivide it into sets of 70:30 ratios, where the algorithm is trained with $70 \%$ and the training is validated with the remaining 30\%. Some algorithms used in this classification problem are: Naive Bayes, Decision trees, Stochastic gradient descent, Logistic regression, Support vector machines (SVM). In [3] taking the results presented in the confusion matrix it can be said that, of the algorithms mentioned above, SVM is the algorithm that shows better results concerning other methods and its data discrimination makes it the most useful to address the type of problem posed.

Recently, new architectures and algorithms for the classification and generation of text have appeared, some of them can be seen in:

- $\quad$ BERT by Google [4]

- $\quad$ GPT by OpenAI [5]

- $\quad$ GPT-2 by OpenAI [6]

- $\quad$ Transformer-XL by Google [7]

- $\quad$ XLNet by Google [8]

- XLM by Facebook [9]

- RoBERTa by Facebook [10]

- DistilBERT by HuggingFaceb [11]

- CTRL by Salesforce [12]

- $\quad$ CamemBERT by Inria, Facebook y Sorbonne [13]

- $\quad$ ALBERT by Google Research and the Toyota Technological Institute at Chicago [14]

\section{SPATIAL FRAMEWORK}

With approximately 8 million inhabitants, Bogotá is one of the most populated cities in Latin America, and due to the limitation of urban land for new construction is one of the most densely populated cities in the world, along with cities such as Calcutta in India or Lagos in Nigeria. This demographic and economic growth is exerting increasing pressure on services associated with mobility [15].

Bogotá's density is close to twenty-one thousand inhabitants per square kilometer and its distribution can be observed in each zonal planning unit (UPZ). An assessment of the number of inhabitants of each UPZ shows that most of the population is concentrated in the western part of the city, leaving the central and eastern areas with fewer inhabitants (Figure 1). Therefore, it is possible to find that some areas of the city are dynamic and during the day the population increases due to the floating population, and decreases during the night mainly due to the location of centers of economic activity, causing the population to make long trips to their places of study or work. 


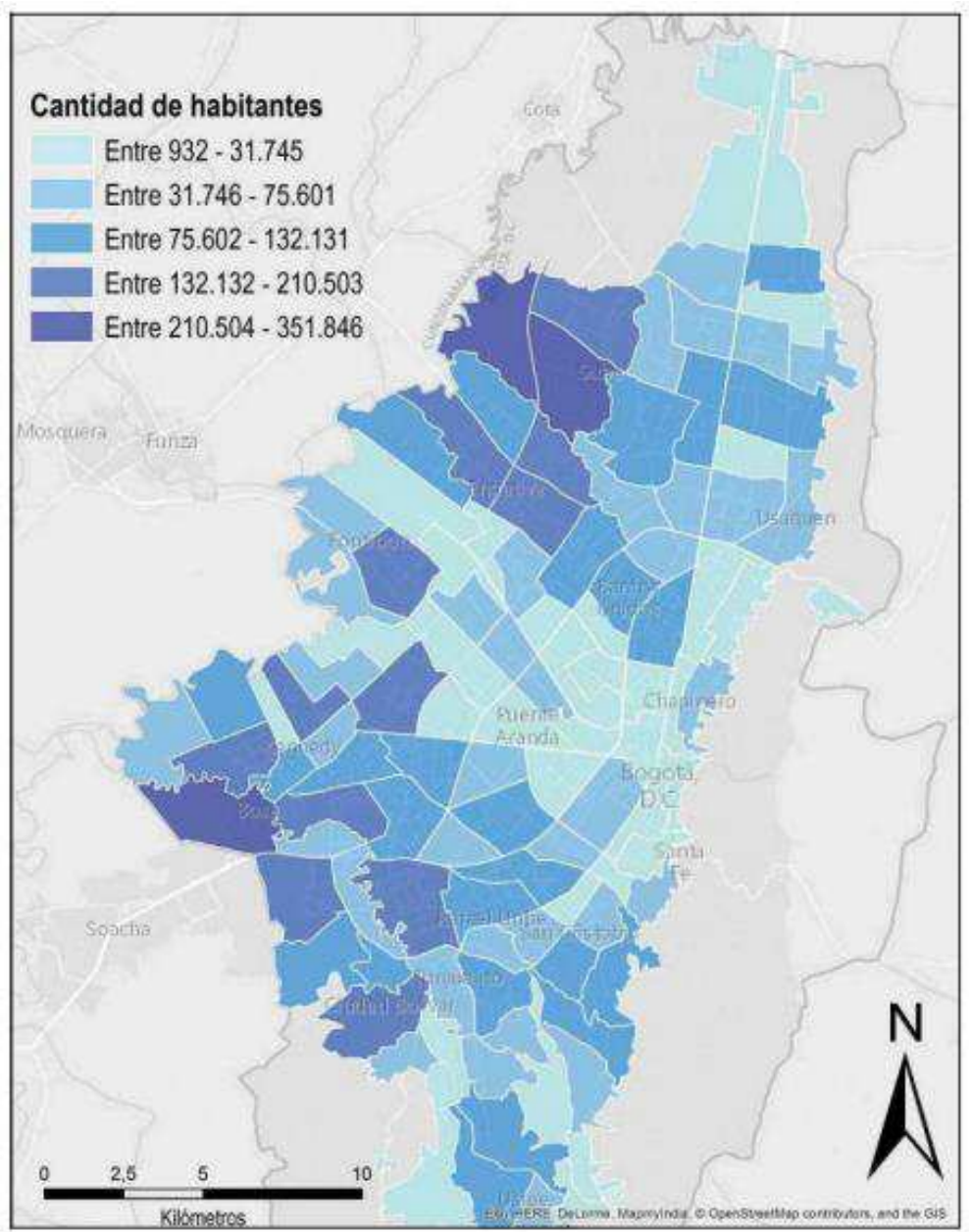

Figure 1: Bogota population map by zonal planning unit - taken from [15]

According to data from the Mobility Observatory and the 2015 mobility survey, the inhabitants of Bogota make $13,325,901$ trips a day, of which $43 \%$ are made by mass and collective public transport, $23 \%$ by a pedestrian, $12 \%$ by private vehicle and $5 \%$ by motorbike, and the estimated average time per trip is 57 minutes. These data are of importance because characterizing this population it is possible to establish where the majority of mobility problems, recurrent and non-recurrent traffic are found.

\section{ROAD INCIDENCE DATA CAPTURE}

There are several ways to acquire information related to transport networks, within these are, active traffic systems that control a key variable that is the speed limit, which is commonly used to improve mobility and reduce traffic accidents. Another type of information is recorded by police entities that report traffic accident data, generating a history with location, type of involved, severity, time and date, combining spatial databases. However, the effectiveness of traditional incident detection is often limited by the limited coverage of sensors on a road mesh and the reporting of incidents to response systems is labor-intensive [16].

In response and to detect incidents cost-effectively in real-time, researchers have used various techniques and methods of artificial intelligence, including: California Method, Discriminatory Statistical Algorithms, Vector Support Machines, Artificial Neural Network, Kalman Filter, Sequential Probability Ratio Test (SPRT), Integrated Moving Average Autoregressive Model (ARIMA), High Occupancy Algorithm (HIOCC), wavelet models and Clusters. Other 
researchers, thinking about the profitability of access to real-time data, have evaluated crowdsourcing as an alternative to data collection, concluding that these data can work in real-time and are also reasonably accurate.

Some ways of identifying traffic patterns have been implemented more recently; one of these techniques is traditional statistical modeling, such as Bayesian logistic regression from which researchers assumed a linear relationship between dependent and independent variables. Another technique used in the identification of traffic patterns are vector support machines based on the theory of statistical learning, a third technique is used with neural networks, although some authors indicate that they are black boxes [17] others take advantage of them with deep learning.

Finally, enthusiasts have undermined social networks, resulting in research that presents methodologies for tracking, processing, and filtering public tweets in real-time, then analyzed to extract information about traffic incidents through deep learning. [18]

For this work, we have taken a corpus of 2432 tweets, in this case as said in section 2 we make use of the SVM model for its good results in the classification of texts and because their training is supervised we must have a set of data already labeled. Thus, the corpus enters the model labeled in two categories; with the number " 0 " are identified the texts without incidence on the road and with the number " 1 " those text chains that report an incidence on the road, understanding by incidence: fall of a stationary vehicle, a motorcyclist or a bicycle, collision, collision with a dead person, traffic light without functioning, fall of a tree, flooding on the road, among others. In this way the training was carried out with 1012 tweets that refer to some type of incidence on the roadand 1420 tweets are not related to events on the roads.

\section{MODEL TRAINING AND TUNING}

As mentioned in the previous section, 2432 tweets in Spanish will be used to identify road incidents in the city of Bogota, this group will be the training data set. The normal text classification process used at this point needs to clean up the information and finally vectorize it. The tasks performed to correspond to the following:

- Tokenize, convert the text string into a list of words and remove punctuation marks.

- Convert all words to lowercase.

- Remove "stopwords", "stopwords" are frequent words that do not add value to the phrases, some examples would be the words "from", "by", "with", among others.

- Stemming, is the process by which words are transformed to their root, for example, "wonderful", "wonder" or "marvel", in this case, will be considered the same word.

With this, it would be enough to run the SVM algorithm with random parameters or search for the best manually, but the objective in machine learning is to search for the best model and parameters, to refine the results the best option is to search for the best hyperparameters for the SVM model [19], instead of searching for the data manually this can be done automatically with the help of "Grid Search", the grid search scans the data to configure optimal parameters for a given model.

Depending on the type of model used, certain parameters are necessary. Grid search can be applied through automatic learning to calculate the best parameters to be used for any given model. It is important to note that grid searching can be extremely computationally expensive and can take a long time to run your machine. Grid-Search will 
create a model in every possible combination of parameters. It runs through each combination of parameters and stores a model for each combination. The best parameters for the model are shown in Table 2.

Table 2: SVM HYPERPARAMETERS with grid-search

\begin{tabular}{|l|l|}
\hline HYPERPARAMETERS & valor \\
\hline C & 0,2 \\
\hline Loss & Hinge \\
\hline Max_iter & 1000 \\
\hline Max_df & 1,9 \\
\hline Max_features & 500 \\
\hline Min_df & 10 \\
\hline Ngram_range & $(1,2)$ \\
\hline
\end{tabular}

The hyperparameters in table 1, the most significant value is c, a small value is more permissive with values that do not respect the classification margin, this can be seen in Figure 2, whereas the margin is small allows some individuals to cross the plane of separation, the other parameters refer to the number of iterations and other parameters of the library used.

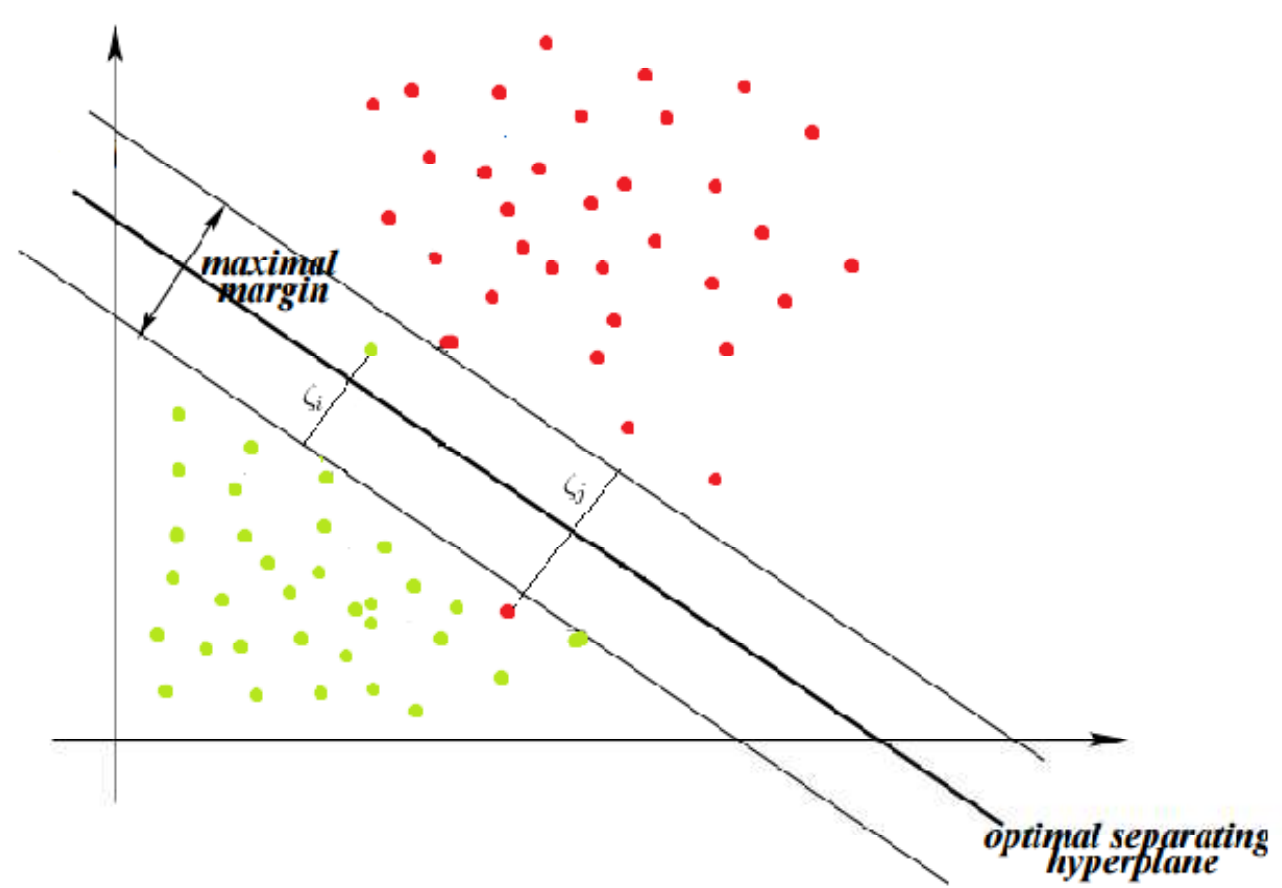

Figure 2: Slack variable

The evaluation of the classifier's performance was carried out using the confusion matrix. The confusion matrix for the trained model can be seen in Table 3 . 
Table 3: Confusión matrix

\begin{tabular}{|l|l|l|l|}
\hline \multicolumn{3}{|l|}{ Predict class } \\
\hline \multirow{4}{*}{ Actual class } & & 0 & 1 \\
\cline { 2 - 4 } & 0 & 900 & 520 \\
\cline { 2 - 4 } & 1 & 582 & 530 \\
\hline
\end{tabular}

The matrix shows the classification, the first row considers texts in which there were no road incidents, 900 correctly classified and called true negatives, while 520 were erroneously classified as road incidents (false positives), the second row considers texts containing road incidents were erroneously classified as 582 (false negatives), while 530 were classified as true positives.

For the next section, 27,194 new social network texts were captured so the model is run to classify. From the classification, 12,580 were categorized as incidences in the arterial roads of the city these were migrated to the process of extraction of geographical context.

\section{NAMED ENTITY EXTRACTION (NER) AND GEOGRAPHIC INFORMATION RETRIEVAL (GIR)}

The objective is to extract spatial data from the information in the texts, and thus know in which direction or geographical location a road incident is located, which makes it necessary to know the type of named entities that are to be extracted. For example, if it is required to know that "https://www.udistrital.edu.co" is a URL, then the patterns that make up a URL must be identified and specified to be recognized. One of the options generally used is to make extraction with a large corpus of information, it is common when it comes to extracting information that works use Wikipedia to extract entities. [20]

In addition to recognizing names and dates in a text, it is possible to extract general information about it using NER training models that allow you to find connections between the information, however, you can also find patterns in the writing and establish them with algorithms using regular expressions. A regular expression is defined by a finite number of states in such a way that the text can enter a decision tree that evaluates a sequence of symbols such as ASCII character strings or a sequence of words, also called grammar[21].

Geographic Information Retrieval (GIR) systems or geographic focus (a place or places where the content of a text is centered) are specialized from traditional information retrieval systems focused on obtaining documents relevant to a given location [22]. It is common to find works that once make recognition of named entities and their geographical context attempt later disambiguation of toponyms, to specify which among multiple localizations a text can be associated. For example, in Colombia, all cities have a main square or park with the name "Simón Bolívar", so it is necessary to discriminate to which place in the space a text string refers, this can be seen in [23]-[25]. Other researchers have faced the challenge of classification and disambiguation with the help of different data corpus such as GeoNames to build scalable frameworks such as Geotxt [2] and predesigned NER implementations in different libraries.

For this work, given the scale, urban, regular expressions were used on the directions of the city of Bogota, where the main road is reported on one side and then the secondary road as shown in Table 4. 
Table 4: Recovery Target

\begin{tabular}{|l|l|l|}
\hline No. & Main road & Secondary road \\
\hline 1 & Av. Caracas & Calle 19 \\
\hline 2 & Av. Circunvalar & Calle 16 \\
\hline 3 & Carrera 60 & Av. Esperanza \\
\hline
\end{tabular}

\section{GEOCODING AND IDENTIFICATION OF SPATIAL PATTERNS}

From the total number of tweets classified as road incidents, a total of 7,169 were extracted with a geographical context, representing $56.98 \%$ of the initial classification. Figure 3 shows the location of these events.

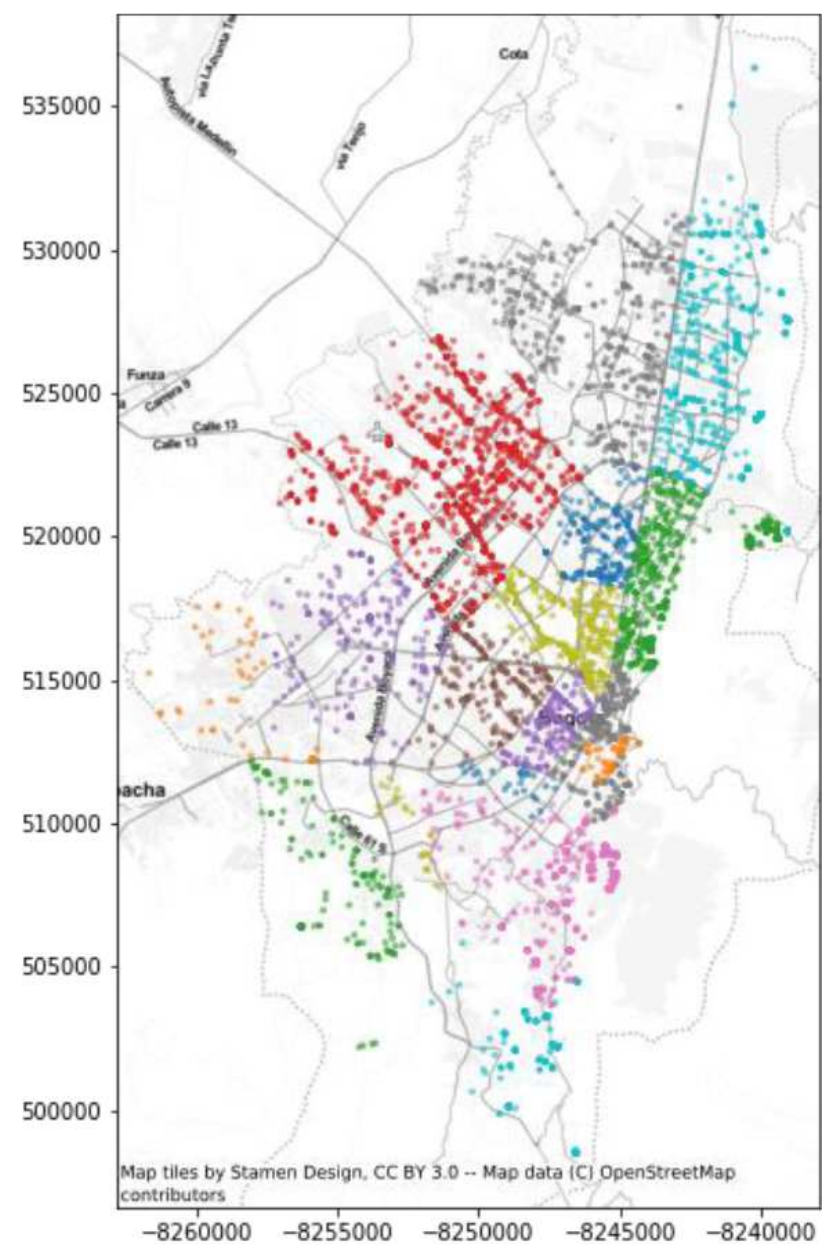

Figure 3: Spatial distribution of road incidence data

Figure 3 shows the distribution of the events but does not allow us to visualize a pattern in the data, for which the potential of the raster format is used and a heat map is obtained in Figure 4 that allows us to identify those zones more prone to accumulate road incidences. 


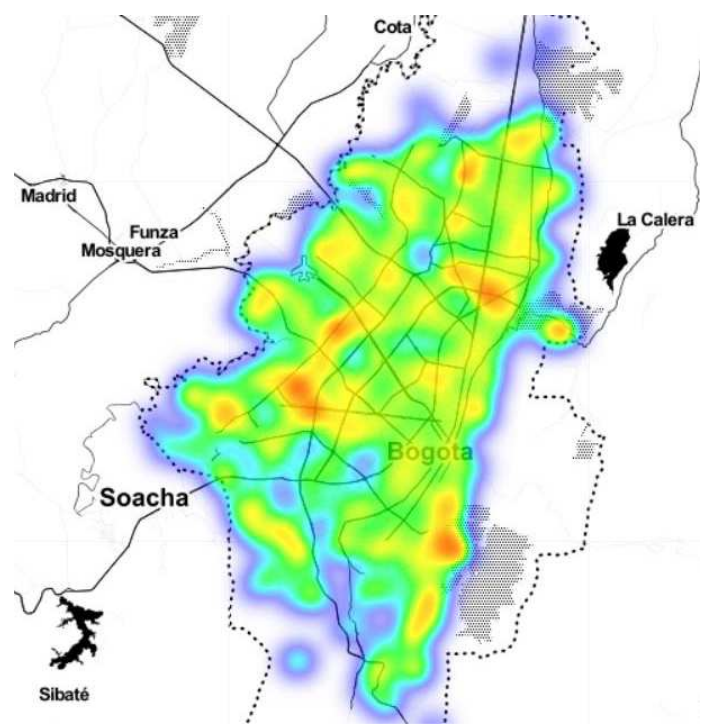

Figure 4: Heat map of road incidence data

Patterns can be seen in the spatial distribution of the data, some localities such as Kennedy, Ciudad Bolívar and Chapinero are those with high heat zones and the number of events per search range is greater. In Figure 5 an analysis can be seen from the number of times the text was shared, this is done with hot-spot but weighted by the importance of tweets indicating in this way the areas where the help of the road control system or traffic police is evident, as shown below.

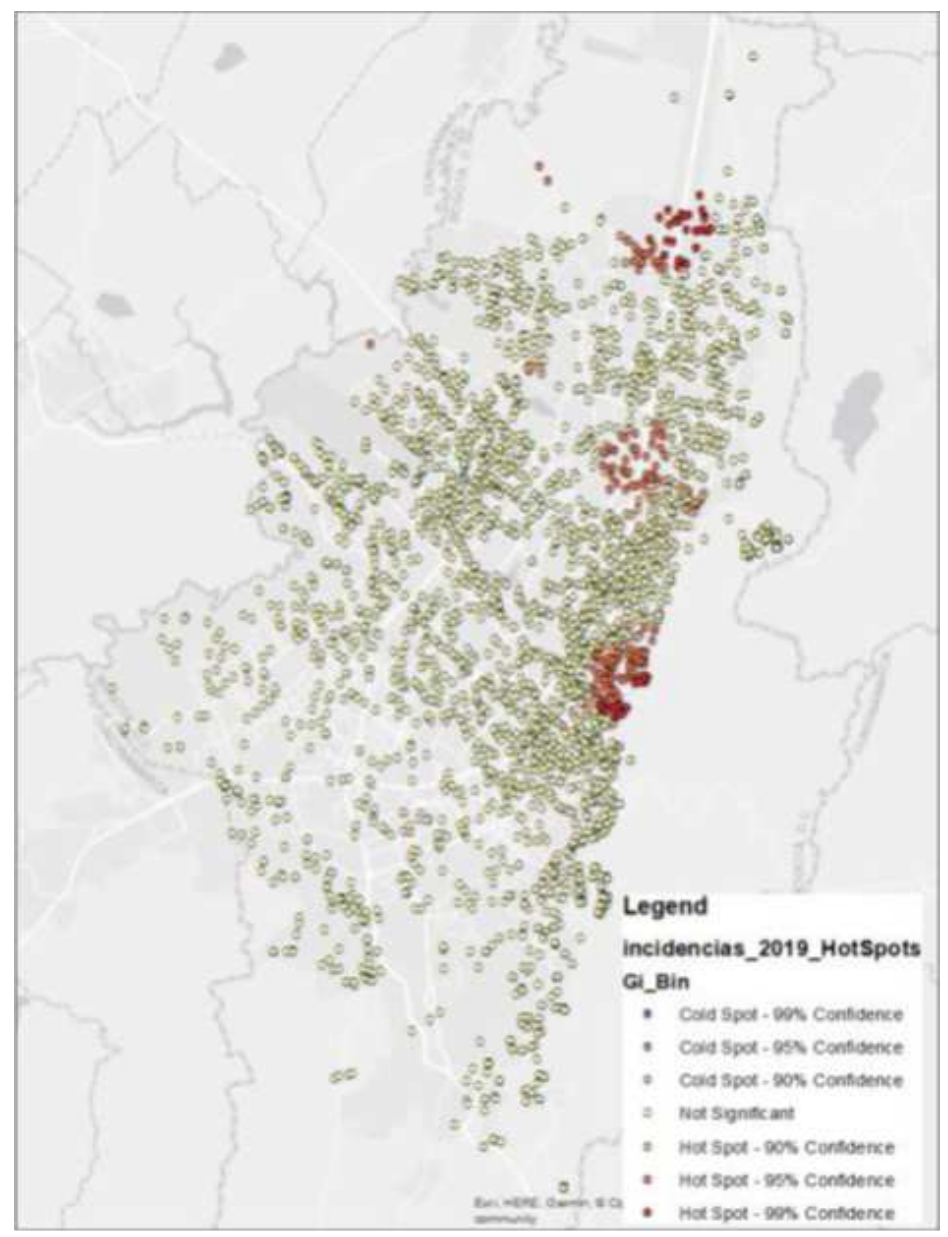

Figure 5: HotSpots analysis of road incidence data 


\section{CONCLUSIONS}

The contribution of this work is focused on the extraction of spatial information and its application in the identification of patterns, it is a challenge for the field of artificial intelligence to achieve better classifications of events and the correct extraction of information, between that recovery, geographical contexts are especially difficult to find because there are many topological ambiguities, to this is added that the texts studied are short and the context that can be extracted is limited.

The method of regular expressions for a problem of the scale of detail, in this case, was the best, because it leaves aside the ambiguity of topological names and focuses on encodings generated within the city, such as names and number of way, the recovery of coordinates does not exceed $60 \%$ but is a breakthrough in working with data as detailed as incidents on away.

GIR systems are evolving continuously, there is no longer talk of recovery models and systems, which means having several networks of classification and extraction. The field of study allows many possibilities for future work such as the management of toponyms, the generation of the giant corpus or geographical dictionaries, classification with an information retrieval system and complementing its extraction with regular language patterns.

It is evident that spatialized data for the case example, road incidences, there are patterns and demonstrates the enormous potential that has the unstructured data at present for the management of the city in real-time. The adoption of these techniques makes monitoring simpler with less labor and less technological infrastructure on the road network.

\section{ACKNOWLEDGMENTS}

The authors would like to thank the Universidad Distrital Francisco José de Caldas and the LASER research group that supported the development and testing of the project.

\section{REFERENCES}

1. Chen, P-T., Chen, F. \& Qian, Z. (2014). Road Traffic Congestion Monitoring in Social Media with Hinge-Loss Markov Random Fields. In Proceedings 2014 IEEE International Conference on Data Mining (ICDM). p.p. 8089.

2. Karimzadeh, M., Pezanowski, S., MacEachren, A. M. \&Wallgrün, J. O. (2019). GeoTxt: A scalable geoparsing system for unstructured text geolocation. Transactions in GIS. 2019. 23(1):118-136.

3. Hardeniya, N., Perkins, J., Chopra, D., Joshi, N. \&Mathur, I. (2016). Natural Language Processing: Python and NLTK. Packt Publishing Ltd; 2016.

4. Devlin, J., Chang, M-W., Lee, K. \&Toutanova, K. 82019). BERT: Pre-training of Deep Bidirectional Transformers for Language Understanding. In Proceedings of NAACL-HLT 2019, Minneapolis, Minnesota, June 2 - June 7, 2019 p.p. 4171-4186.

5. Radford, A., Narasimhan, K., Salimans, T. \&Sutskever, I. (2018). Improving Language Understanding with Unsupervised Learning. Retrievedfrom: https://cdn.openai.com/research-covers/languageunsupervised/language understanding_paper.pdf

6. 
7. Radford, A., Wu, J., Child, R., Luan, D., Amodei, D. \&Sutskever, I. (2019). LanguageModels are Unsupervised Multitask Learners. Retrievedfrom: https://cdn.openai.com/better-languagemodels/language_models_are_unsupervised_multitask_learners.pdf

8. Dai, Z., Yang, Z., Yang, Y., Carbonell, J., Le, Q. \&Salakhutdinov, R. (2019). Transformer-XL: Attentive Language Models beyond a Fixed-Length Context. In Proceedings of the 57th Annual Meeting of the Association for Computational Linguistics. 2019.

9. Yang, Z., Dai, Z., Yang, Y., Carbonell, J., Salakhutdinov, R. \& Le, Q. (2019) XLNet: GeneralizedAutoregressivePretraining for Language Understanding. 33rd Conference on Neural Information Processing Systems (NeurIPS 2019), Vancouver, Canadá.

10. Conneau, A. \&Lample, G. (2019). Cross-lingual Language Model Pretraining. 33rd Conference on Neural Information Processing Systems (NeurIPS 2019), Vancouver, Canadá.

11. Liu, Y., Ott, M., Goyal, N., Du, J., Joshi, M., Chen, D., Levy, O., Lewis, M., Zettlemoyer, L. \&Stoyanov, V. (2020).RoBERTa: A Robustly Optimized BERT Pretraining Approach.ICLR 2020 Conference.

12. Sanh, V., Debut, L., Chaumond, J. \&Wolf, T. (2019). DistilBERT, a distilled version of BERT: smaller, faster, cheaper and lighter. NeurIPS 2019 workshop.

13. Keskar, N. S., McCann, B., Varshney, l. R., Xiong, C. \&Socher, R. (2019). CTRL: A Conditional Transformer Language Model for Controllable Generation. Retrieved from: https://arxiv.org/abs/1909.05858v1

14. Martin, L., Muller, B., Ortiz Suárez, P. J., Dupont, Y., Romary, L., Villemonte de la Clergerie, E., Seddah, D. \&Sagot, S. (2020). CamemBERT: a Tasty French Language Model. Retrievedfrom: https://arxiv.org/abs/1911.03894v1

15. Lan, Z., Chen, M., Goodman, S., Gimpel, K., Sharma, P. \&Soricut, R. (2020). ALBERT: A Lite BERT for Selfsupervised Learning of Language Representations. Retrievedfrom:https://arxiv.org/abs/1909.11942

16. Secretaria Distrital de Movilidad. (2017). Observatorio de movilidad Bogotá D.C 2017. Retrievedfrom:http://paperpile.com/b/tekOMX/3q3I

17. Gu, Y., Qian, Z. \& Chen, F. (2016). From Twitter to detector: Real-time traffic incident detection using social media data. Transportation Research Part C: Emerging Technologies. 67:321-342.

18. Yu, R. \& Abdel-Aty, M. (2013). Utilizing support vector machine in real-time crash risk evaluation. Accident Analysis \& Prevention. 51:252-259.

19. Zhang, Z., He, Q., Gao, J. \& Ni, M. (2018). A deep learning approach for detecting traffic accidents from social media data. TransportationResearchPart C: Emerging Technologies. 86:580-596.

20. Géron, A. (2017). Hands-On Machine LearningwithScikit-Learn and TensorFlow: Concepts, Tools, and Techniques to Build Intelligent Systems. “O’Reilly Media, Inc.”; 2017.

21. Lane, H., Hapke, H. \& Howard, C. (2019). Natural Language Processing in Action: Understanding, Analyzing, and Generating Text with Python. Manning Publications Company; 2019. 
22. Zhang, Z., Ni, M., Gao, J. \&He, Q. (2016). Mining Transportation Information from Social Media for Planned and Unplanned Events. Final Report. University at Buffalo, SUNY. Retrieved from:https://rosap.ntl.bts.gov/view/dot/30838

23. Peregrino, F. S., Díaz, D. T. \& Llopis, F. (2013). Una aproximación basada en corpus para la detección del foco geográfico en el texto. Procesamiento del lenguaje natural. 50:69-76.

24. Leidner, J. L. (2008). ToponymResolution in Text: Annotation, Evaluation and Applications of Spatial Grounding of Place Names. Universal-Publishers; 2008.

25. Martins, B. \& Silva, M. J. (2005). A Graph-Ranking Algorithm for Geo-Referencing Documents. Fifth IEEE International Conference on Data Mining (ICDM’05).

26. Anastácio, I., Martins, B. \& Calado, P. (2009). Classifying Documents According to Locational Relevance. Progress in Artificial Intelligence. Progress in Artificial Intelligence (EPIA 2009). pp. 598-609. 